\title{
Presencia de la Generación del 27 en la revista $C A L$ (Sevilla, 1974-1979): un diálogo intergeneracional
}

\author{
The Generation of 1927 in the literary journal Cal (Seville, 1974-1979): an \\ intergenerational dialogue
}

José Jurado Morales

jose.jurado@uca.es

Universidad de Cádiz, España

Recepción: 12 Agosto 2019

Aprobación: 05 Mayo 2020

Publicación: 15 Mayo 2020

Cita sugerida: Jurado Morales, J. (2020). Presencia de la Generación del 27 en la revista $C A L$ (Sevilla, 1974-1979): un diálogo intergeneracional. Olivar, 20(31), e076. https://doi.org/10.24215/18524478e076
Resumen: En este artículo se estudia el propósito de Joaquín Márquez, director de la revista Cal (Sevilla, 1974-1979), y sus compañeros de abrir un diálogo intergeneracional en los tiempos de la Transición política de los años setenta mediante la publicación de textos de algunos escritores veteranos que contribuyen a enlazar el pasado y el presente poético de Sevilla y, en general, de Andalucía. Desde esta óptica se analiza la incorporación de poetas próximos a la Generación del 27 a la revista $\mathrm{Cal}$. Se da cuenta de los números especiales dedicados a Rafael Laffón, Luis Cernuda, Vicente Aleixandre y los poetas sevillanos, y se comentan los textos de los poetas del núcleo central del 27 incluidos en Cal: Aleixandre, Cernuda, Diego y Guillén, al igual que los de otros coetáneos que inician su trayectoria en la Edad de Plata: Sierra, Laffón, Pemán, Barbadillo, Jurado Morales, Ruiz Peña, Garciasol, Prieto, Cano, Vivaldi, GilAlbert, Conde y Lagos.

Palabras clave: Joaquín Márquez, Poesía española contemporánea, Transición, Generación del 27.

Abstract: This article studies one important aspect of the Seville-based literary journal Cal. It proposes that the aim of Joaquín Márquez —Cal's general editor- and his collaborators was to open up an intergenerational dialogue in the times of the Spanish political transition of the 1970s. Such a dialogue was made effective through the publication of the work of a number of veteran writers who contributed to link the poetic past and present of Seville and, by extension, of Andalusia. From that perspective, it is analysed the incorporation to the pages of Cal of poets close to the Generation of 1927. Particular consideration is given here to the special issues of the journal devoted to the relationship between Rafael Laffón, Luis Cernuda, Vicente Aleixandre and the Sevillian poets, as well as to the texts produced by some of the principal authors of such generation (Aleixandre, Cernuda, Diego and Guillén) and by some of their contemporaries (Sierra, Laffón, Pemán, Barbadillo, Jurado Morales, Ruiz Peña, Garciasol, Prieto, Cano, Vivaldi, Gil-Albert, Conde, and Lagos).

Keywords: Cal literary journal, Joaquín Márquez, Contemporary Spanish poetry, Transition, Generation of 1927. 


\section{La Revista Cal en la Sevilla poÉtica de los años 701}

El precedente de la revista Cal se halla en el ámbito del grupo Ángaro, que surge el 15 de enero de 1968 con las reuniones literarias dirigidas por el poeta y sacerdote leonés Manuel Fernández Calvo en el Bodegón Torre del Oro de Sevilla. De aquel embrión surgen unas entregas de poesía, una colección y, a partir de 1970, un premio anual de nombre homónimo al grupo (Ruiz Copete, 1983, p. 55; Cruz Giráldez, 1994; Cenizo Jiménez, 2002, p. 204; Simón Guzmán, 2008, 2015). También emerge de esas tertulias la idea de publicar una revista, que finalmente se estampa con el título de Cal. Revista de poesía y que pervive entre enero de 1974 y noviembre de 1979 con una periodicidad bimensual y bajo la dirección de Joaquín Márquez. La publicación tiene una primera etapa hasta 1978 en la que se publican treinta números con Onofre Rojano como secretario y administrador, y una segunda, que consta de seis números, en la que figuran Enrique Molina Campos como subdirector y Ángel Crespo como consejero.

La salida de Cal supone un aliciente en el ámbito cultural y la reanudación de una actividad poética más vigorosa en la Sevilla de los años sesenta después de un periodo en el que la vida poética merma en la ciudad. Los poetas sevillanos más renombrados del medio siglo, que pudieran hacer las veces de referentes y guías de los más jóvenes, andan fuera de la ciudad: Rafael Montesinos reside en Madrid desde comienzos de los cuarenta; Julia Uceda se marcha a los EE.UU. a mediados de los sesenta y se establece en Irlanda en los setenta; Manuel Mantero se afinca en Madrid al comienzo de la década de los sesenta y a finales de la misma se instala en EE.UU.; Aquilino Duque no para de viajar de un lugar para otro y se afinca en Ginebra y Roma en los sesenta y setenta; Manuel García-Viñó vive en Madrid desde antes de comenzar los sesenta (Cózar, 1977; Ruiz-Copete, 1971, p. 359). Casi solo Joaquín Caro Romero salva Sevilla de tanta desolación poética (RuizCopete, 1971, pp. 335-345). Otro tanto puede comentarse al respecto de las revistas sevillanas, en un tiempo significativas y en estas fechas inexistentes. Asimismo, desde finales de los años setenta ganan en popularidad los narradores, los llamados narraluces, frente a los poetas. Ruiz Copete lo explica así:

En el lustro comprendido entre 1960 y 1965 [...] se produce un bache poético no sólo por la ausencia de una nueva calidad sino también y, sobre todo, por la falta numérica de poetas de veinte a veinticinco años que asegura la continuidad del legado poético en esa hora. [...] Mas bastaba una mera prospección del panorama para advertir, en efecto, la ausencia de nombres nuevos, incluso en aspectos como el de las revistas o los grupos poéticos que en muy pocos años anteriores habían sido no sólo numerosos sino que aportaron una indudable calidad. (Ruiz Copete, 1983. p. 48)

De modo que, con la clara conciencia de que Cal pretende reactivar la vida poética sevillana, Joaquín Márquez presenta un texto de apertura del primer número titulado "Pórtico", donde expresa el sentir común sobre el vacío y el desnortamiento poético de la Sevilla de la época y el deseo de aunar esfuerzos y recuperar el interés de los lectores:

Grecia, Mediodía, Guadalquivir, Aljibe, Ixbiliah, Rocío, Loreley... Largo número de revistas de poesía que, con más o menos fortuna, han levantado el estandarte poético de nuestra ciudad. Y después el silencio. Un silencio que dura ya veinte años.

¿Qué ocurre en esta tierra de líricos entrañables para que no prevalezcan, unidas en el esfuerzo, las voces de sus cantores? La independencia de nuestros poetas, la confusión de los estilos y el caos -¿̨por qué no decirlo?- de experiencias y juegos seudopoéticos, han trabajado en el tiempo contra la poesía, han ido separando la tierra de las raíces, y han enredado en la maraña de la duda y el desinterés al lector y al propio poeta.

¿Cuántos poetas han ido creciendo, madurando, bajo este silencio?

Ahora nace una revista para intentar averiguarlo, para gritar "no" a las voces que anticipadamente lloran la muerte de nuestra poesía. Una revista abierta a todos los poetas y que pretende sólo dar a conocer, desterrando prejuicios, la poesía en cualquier forma que se presente.

Los que hoy fundamos la revista $C A L$, queremos levantar una pared blanca donde el poeta pueda dibujar sueños. Una pared alta que sostenga su firmamento de inquietudes, alegrías y tristezas. Una pared de cal donde apretar los huesos contra el cansancio. Una blanca y alta pared encalada, donde pueda ir dejando su oscura señal el hombre que pasa. (Márquez, 1974, p. 2) 
El afán programático por unir esfuerzos de Márquez explica el sentido conciliador de Cal, que acoge poetas diversos que se mueven por las colecciones sevillanas Ángaro, Barro, Aldebarán y Dendrónoma así como otros escritores foráneos (Cenizo Jiménez, 2002, p. 204). También explica la presencia de poetas pertenecientes a generaciones diferentes: hay escritores del 27, de la primera posguerra, del medio siglo, de los sesenta y de los años de la Transición. En las palabras citadas de su director, consiste en "Una revista abierta a todos los poetas", sin prejuicios y dispuesta para "la poesía en cualquier forma que se presente". Joaquín Márquez propone, pues, un posicionamiento plural e integrador de estéticas, un manifiesto en favor de la heterogeneidad de poetas y poéticas.

Este sentido conciliador, intergeneracional y pluriestético supone un mérito para algunos críticos, que han valorado positivamente la iniciativa de Márquez y el grupo Cal en la reactivación poética de Sevilla, y un demérito para otros críticos, que subrayan la disparidad de estéticas, la calidad irregular de sus poemas y la poca repercusión que tiene la revista (Jurado López, 1980, 1989, 1994; Ruiz Noguera, 1989; Rodríguez Pacheco y Sánchez Menéndez, 1992; Jiménez Martos, 1978; Cenizo Jiménez, 2002; Ruiz Copete, 1983; Barrera, 1992; Cruz Giráldez, 1994). Joaquín Márquez remite a estas críticas en un texto sin título que abre el número 30 de Cal en noviembre de 1978:

Durante este tiempo hubo críticas adversas \# ¿qué obra humana que se precie no las ha tenido?\#, pero, afortunadamente, fueron muchas más las voces que nos alentaron en esta labor desinteresada donde nombres importantes y nombres que aún no lo son, pero pueden llegar a serlo, se mezclaron poniendo sus granos en la arena poética de nuestra obra. Sin embargo, no es hora de hacer un balance porque esta pared no va a derribarse. Sólo vamos a dar un paso adelante. (Márquez, 1978b, p. 2)

He citado esta declaración de noviembre de 1978 porque entiendo que alude -de forma elíptica y probablemente no de forma exclusiva- a un debate acalorado e intenso entre el propio Joaquín Márquez y el librero y poeta Abelardo Linares, coeditor por entonces de la revista Calle del Aire, en las páginas del semanario Tierras del Sur en un número de setiembre de ese 1978. Ahí figuran las opiniones de uno y otro bajo el titular común de "Una de Cal y otra de Aire". Abelardo Linares le afea "mezclar indiscriminadamente los nombres", "la falta de un criterio de selección" de poetas y la ingenuidad o demagogia de pretender llegar a la generalidad del pueblo andaluz (Linares, 1978, p. 53). Márquez argumenta en defensa de Cal la diversidad y calidad de los colaboradores, la difusión de la revista con más de diez mil ejemplares tirados y la supervivencia durante cuatro años con una subvención precaria (Márquez, 1978a, p. 52). Abelardo Linares le señala esa mezcla y disparidad estética en estos términos:

Con ello no hace sino servirme el mejor argumento contra su revista. Y es éste: la falta de un criterio de selección. Mezclar indiscriminadamente los nombres de Jorge Guillén y Juan Cervera, Vicente Aleixandre y Joaquín Benito de Lucas -por solo citar unos cuantos-, o es desinformada buena intención o simple despropósito.

¿Cuál es el criterio, la visión de la poesía de una revista en la que, en torpe mezcolanza, cualquier lector puede encontrar maestros del 27, glorias locales, cultivadores del surrealismo, cantores de provincia, sonetistas de juegos florales, desenfrenados experimentalistas, poetas existenciales, meditativos y sociales y otros mil especímenes inclasificables de aficionados al verso libre y medido? (Linares, 1978, p. 53)

Traigo a colación esta polémica entre Abelardo Linares y Joaquín Márquez porque, en última instancia, nos acerca a la cuestión medular de este trabajo: la presencia de los poetas del 27 en la revista $\mathrm{Cal}$. Como anota Linares, “cualquier lector puede encontrar maestros del 27", lo que habla a las claras del deseo de diálogo intergeneracional y de recuperación de algunas voces veteranas que guía el propósito de Joaquín Márquez y sus compañeros de revista.

\section{Presencia del 27 en la revista Cal}

Si bien abundan los poetas jóvenes, sobre todo en el comienzo de la revista, con el paso de los números el elenco de Cal se abre a gente más madura, cuya trayectoria arranca en la primera posguerra, y a maestros más experimentados, que inician su andadura en plena Edad de Plata en la órbita de los poetas del 
27. Un repaso por los índices de Cal nos revela la participación de Vicente Aleixandre, Rafael Laffón, Luis Cernuda, Gerardo Diego, Jorge Guillén y Juan Sierra, además de otros más o menos coetáneos como José María Pemán, Manuel Barbadillo, José Jurado Morales, Juan Ruiz Peña, Ramón de Garciasol, Gregorio Prieto, José Luis Cano, Juan Gil-Albert, Carmen Conde y Concha Lagos. Esto evidencia el propósito de Joaquín Márquez y sus colaboradores en abrir un diálogo intergeneracional que sirva de hilo conductor cronológico y geográfico entre el pasado y el presente poético de Sevilla y, en general, de Andalucía.

En breve, volveré a los poemas que estos publican, pero antes quiero recalar en otro aspecto que también vincula la poesía joven de los setenta con la de los referentes del 27. En Cal encontramos un conjunto de artículos de crítica literaria más extensos firmados por Enrique Molina Campos a partir del número 18 de Cal, de enero de 1977. En ellos se centra con preferencia en ofrecer un panorama crítico de la poesía andaluza de los años setenta, pero a veces, en su intento de presentar el devenir de la poesía andaluza contemporánea, hay referencias al 27 en esos artículos. Por ejemplo, en esta línea se sitúan los artículos “Cernuda en Hölderlin” (Molina Campos, 1977), "Dos grandes poetisas andaluzas: Elena [Martín Vivaldi] y Julia [Uceda]" (Molina Campos, 1978) y "Los poetas de 'Silene' y la poesía de José Gutiérrez" (Molina Campos, 1979), donde emparenta las colecciones "Silene” y "Ánade” de Granada con la poesía elegíaca de Hölderlin, Cernuda y Brines. También Manuel Jurado López firma un texto titulado "Linealidad poética entre Mallarmé, Cernuda y Juan Bernier" (Jurado López, 1979).

Con todo, lo más suculento de Cal con respecto al 27 viene dado por la aparición de un número extraordinario dedicado a los poetas sevillanos y de tres números doble en los que se rinde homenaje a poetas sevillanos del 27: Rafael Laffón, Luis Cernuda y Vicente Aleixandre. Doy paso ahora al comentario de estos números siguiendo el orden cronológico de su salida en busca de la presencia del 27 en Cal.

\section{El homenaje a RAFAel LAFFón}

En mayo-julio de 1975 sale el número 9 de Cal con la indicación de “(doble especial)” y el rótulo en su cubierta de "homenaje a Rafael Laffón”. Participa una veintena de escritores: Alberto Álvarez-Ruz, Fernando Allué y Morer, Manuel Barbadillo, Juan Bautista Bertrán, Alfonso Canales, Gerardo Diego, Manuel Fernández Calvo, Ramón de Garciasol, Ángel García López, Luis Jiménez Martos, José Jurado Morales, José Ledesma Criado, Joaquín Márquez, Rafael Montesinos, Rafael Morales, José Félix Navarro, Pedro Perdomo Acedo, Carlos Rodríguez-Spiteri, Juan Ruiz Peña, Francisco Salgueiro, José Luis Tejada y Francisco Toledano.

Abre el número un texto breve de José Luis Núñez titulado "Razón del Homenaje", donde valora el hecho de que Laffón, al que denomina "Espiga aún en pie de aquella inolvidable floresta creadora del veintisiete" (Núñez, 1975, p. 3), no abandonase Sevilla en busca de la gloria de la capital. Justo por no haberse marchado y por haber apoyado a cuantos escritores se le acercan, lo considera "el celoso y sempiterno guía poético del Sur" (Núñez, 1975, p. 2). Así se expresa Núñez:

Laffón fue de los pocos que se atrevieron a quemar las naves de la ilusión. Que él no quiso partir -¿fue necesario?- cuando los otros, amigos de generación y entusiasmo, remontando el torrente escarpado de Despeñaperros, diéronse de bruces con la medusa de infinitos brazos que era -y será, ¿hasta cuándo? - la sirena de agua dulce del centralismo. (Núñez, 1975: p.2)

Cierra su texto con una reflexión sobre por qué no han recogido ningún poema de sus coetáneos:

hemos prescindido de cualquier representatividad coetánea a la hora de confeccionar este homenaje, pensando que quizás sus dignos embajadores, por esta indulgencia propia que da la convivencia cuando se viaja en un mismo convoy generacional -muchos, como el poeta, pueden estar cumpliendo sus bodas de diamante con la vida-, no estén para estos trotes laudatorios, cegados, tal vez por la luz permanente y la estrechez del compartimento. Queden, pues, para los menos afectados por la miopía cronológica, estos menesteres, que el júbilo y el agradecimiento - ¿por qué no?- hacen menos egoístas y quién sabe si más objetivos. (Núñez, 1975, p. 3) 
Sigue una breve consideración de María de los Reyes Fuentes -recuperada de un homenaje a Laffón en la primavera de 1960 (AA.VV., 1960), en honor a su trayectoria y al Premio Nacional de Literatura "José Antonio Primo de Rivera” 1959 en la modalidad de poesía por su antología La rama ingrata-, en la que acentúa algunas notas presentes en su escritura: la unión de lo popular con lo culto, lo vital con lo intelectual, lo humano con lo artístico (Fuentes, 1975, p. 4).

Tras estos dos textos laudatorios en prosa, figura el poema "Primavera de Rafael Laffón” de Gerardo Diego, también recuperado del homenaje de 1960 (Diego, 1975, p. 5) y recogido en la Segunda antología de sus versos (Diego, 1967), donde se sirve de florituras líricas para alabar la escritura del sevillano. Sigue una "Microantolología de Rafael Laffón" con los poemas "Para morir es buena cualquier hora" y "Resurrección", de Vigilia del jazmin, de 1952, y "Madrigal a una linda pecosa" y "Olor de mi madre", de La cicatrizy el reino, de 1954 (Laffón, 1975, p. 6-10). Se cierra el número con una breve noticia bio-bibliográfica de Laffón.

Asimismo, encontramos algunos poemas de escritores coetáneos del grupo del 27 , aunque no formen parte del círculo central ni más afamado y sus primeras publicaciones en formato de libro resulten posteriores a la década de los años veinte. Manuel Barbadillo, nacido en 1891 y cuyo primer libro, Rincón de sol, data de 1936, incluye el poema "A Rafael Laffón”, en el que le hace ofrenda de objetos, realidades y gentes propias del sur (Barbadillo, 1975, p. 13). Ramón de Garciasol, nacido en 1913 y cuyos primeros poemas corresponden a los tiempos de la Guerra Civil, entrega el poema “iSalud, Rafael Laffón!”, donde saluda al homenajeado con tintes humorísticos y existenciales (Garciasol, 1975, p. 18-19). José Jurado Morales, nacido en 1900, se suma con el poema "A Rafael Laffón”, donde deja testimonio de la amistad que comparten (Jurado Morales, 1975, p. 22). Juan Ruiz Peña, nacido en 1915 y cuyo primer poemario publicado es Canto de los dos, de 1940, participa con "Romance del andaluz", de matriz metafísica y existencialista sobre los enigmas de la vida y la muerte (Ruiz Peña, 1975, p. 31).

\section{UNA ANTOlogía de POETAS SEVILlanos}

El número 14-15, de mayo-julio de 1976, ofrece una antología titulada "poetas de Sevilla”. Aquí se incluyen poemas de Vicente Aleixandre, Rafael Laffón y Juan Sierra, entre los integrantes sevillanos del 27, además de otros de Rafael Montesinos, José María Requena, Julia Uceda, María de los Reyes Fuentes, Manuel García-Viñó, Manuel Mantero, Fausto Botello de las Heras, Alberto García Ulecia, Juan Cervera, Emilio Durán Vázquez, Joaquín Márquez, Francisco Vélez Nieto, Joaquín Caro Romero, Manuel Jurado López, José Luis Núñez, Andrés Mirón, Onofre Rojano, José Antonio Moreno Jurado, Alejandro Amusco, Javier Salvago, Abelardo Gil y Carmelo Guillén Acosta.

Joaquín Márquez es el encargado de preparar esta antología con la que se propone mostrar un elenco diacrónico e intergeneracional de los poetas sevillanos comprendidos entre la Generación del 27 y los que se dan a conocer en los años setenta. Por un lado, sin duda esta selección supone un hito mayor y reseñable en la constatación de que Cal ayuda a la revitalización, la integración y la continuidad de la vida poética sevillana. Por otro lado, la proximidad del quincuagésimo aniversario de la reunión de algunos miembros del 27 en Sevilla en diciembre de 1927 con motivo del homenaje a Luis de Góngora influye en el impulso último de Márquez para llevar a cabo esta iniciativa recopilatoria e integradora. En un texto previo de una página titulado "Poetas de Sevilla" Márquez explica lo siguiente:

$C A L$, revista de poesía abierta a todos los poetas de lengua hispana, vuelve a su origen, en este número, para ofrecer la muestra de una poesía hecha por hombres que nacieron en este lugar del Sur.

Cincuenta años separan -en el orden cronológico que sustituye al habitual alfabético de nuestra revista- unas voces de otras. Todas, sin embargo, dan fe de su vigencia creadora en los originales inéditos que presentan. Una excepción honrosa -la del maestro Rafael Laffón-, confirma la norma por la que nos hemos regido para la inclusión de originales. (Márquez, 1976, p. 2)

En esta pared común -cuando las paredes comunes suelen aceptarse con humildad demasiado tarde- aparecen los nombres consagrados y los recién nacidos al verso, unidos por el mortero y la argamasa de la primera tierra: hilo conductor que, pasando 
por Bécquer, Manuel y Antonio Machado, Luis Cernuda y tantos otros, dibuja la historia poética de nuestra ciudad. Poetas que nacieron aquí y que aquí viven, o que amplían los límites de esta tierra por otras latitudes, ocupan las páginas de esta, que sabemos, incompleta antología. (Márquez, 1976, p. 2)

Vicente Aleixandre abre el número dedicado a los poetas sevillanos con el poema autógrafo "Retorno", recogido en los Poemas de la consumación, que tiene aire metafísico al considerar la vida y la muerte, la experiencia, la juventud, el conocimiento y el amor (Aleixandre, 1976, p. 3). Sigue Rafael Laffón con el poema "Ni línea, ni color...", un madrigal de ocho versos en los que elogia la sonrisa y la gracia de sonreír de alguien perteneciente al libro Romances y madrigales, de 1944 (Laffón, 1976, p. 4). La contribución de Juan Sierra se titula "Canción de la mujer pública" y consiste en un poema extenso con versos libres, versículos muchos de ellos, en torno a una prostituta (Sierra, 1976, p. 5).

\title{
Ante el olvido de Sevilla, un recuerdo para Luis Cernuda
}

En noviembre de 1977 sale el número doble 23-24 de Cal bajo el título de "Homenage [sic] a Luis Cernuda”. En unas palabras previas tituladas “1977” se presenta un texto breve -sin firma; interpreto que escrito por Joaquín Márquez- en el que se aportan las razones del recuerdo. Dice así:

\begin{abstract}
En 1977 se cumple el 50 aniversario de la "generación del 27”. Esto, por sí solo, sería motivo suficiente para que nuestra revista dedicara un número de homenaje a una de las voces más puras de la generación: Luis Cernuda. Pero también, en este año, se cumplen los setenta y cinco del nacimiento del poeta en nuestra ciudad y los 50 de la publicación de su primer libro Perfil del aire. Sobran, por ello, razones para que la revista $C A L$ ofrezca desde sus menguadas páginas -en número que no en afecto- un humilde monumento de palabras a quien tan olvidado es en su tierra como conocido universalmente.

No hubiéramos podido escoger mejor momento que este, en que otro poeta sevillano \#amigo y paisano en la luz y en el verbo\# ha hecho estallar su nombre ( $i$ Vicente Aleixandre!) ante el asombrado Sur, con toda la dinamita del Nobel.

Desde estas páginas de homenaje a Luis Cernuda, nuestro agradecimiento a cuantos han colaborado en ellas y a los que hubieran colaborado si nuestros medios no estuvieran por debajo de nuestros deseos y, especialmente, a Ángel María Yanguas Cernuda \#sobrino del poeta\# que puso a nuestra disposición originales, fotografías y sus mejores atenciones. A él y a todos, gracias.
\end{abstract}

Este pórtico de la revista justifica el homenaje: desde un punto de vista temporal y geográfico resultan tan oportunos el recordatorio del encuentro de la Generación del 27 en la Sevilla de diciembre de 1927 como la reivindicación de uno de los grandes poetas sevillanos tan olvidado en su tierra natal, como bien atestigua el poema de cierre de la revista, "Sevilla 1977 (calle del aire)", de Joaquín Márquez: "Luis Cernuda ha de quedar / para siempre convencido / que Sevilla es un olvido / al otro lado del mar” (Márquez, 1977, p. 44).

El "Homenage [sic] a Luis Cernuda" se presenta con un dibujo en la cubierta de Gregorio Prieto, el pintor nacido en Valdepeñas tan vinculado a la Generación del 27 y tan unido al poeta sevillano. Prieto y Cernuda se conocen en 1924 en una tertulia celebrada en casa de Concha Albornoz, coinciden en Londres tras el comienzo de la Guerra Civil y consolidan su amistad a partir de 1939 durante sus exilios en el Reino Unido. Prieto pinta unos veintitrés retratos de Cernuda, muchos de ellos incluidos en su libro Cernuda en línea (Prieto, 1981). Precisamente, parte de uno de ellos fechado en 1939 se recoge en la cubierta de Cal. Por su lado, Cernuda dedica dos poemas a Prieto: "Jardín antiguo", fechado en Londres el 23 de noviembre de 1939 - esta es la fecha de la carta en la que Cernuda le envía el poema manuscrito con esta dedicatoria: "a Gregorio, con la vuelta de la juventud"2 - y publicado en Las nubes, y "El amigo", fechado el 24 de diciembre de 1944 \#según reza al pie del poema manuscrito autógrafo: "para Gregorio. Londres, Nochebuena de 1944"3. e incluido en Vivir sin estar viviendo.

Asimismo, este número de Cal contiene varios documentos vistosos y singulares cedidos por su sobrino Ángel María Yanguas Cernuda: la reproducción de una foto de Cernuda sin fechar; la autodedicatoria manuscrita de la primera edición de Perfil del aire publicada como suplemento de Litoral: "A Luis con mi cariño / y mi antipatía de siempre, / Luis / 1 Diciembre, 1932”; la dedicatoria que le hace Federico García 
Lorca de Canciones. 1921-1924, en la edición del primer suplemento de Litoral: "Para Luis / con un abrazo de su / Federico / Noche de baby y Miguel Ángel. / Madrid 1935. / ¡Ay qué gritos se sentían / por encima de las casas!"; y el poema manuscrito "El amigo", dedicado a Gregorio Prieto que acabo de mencionar.

En cuanto a la selección de poemas y prosas se agrupan textos de Narzeo Antino, Alfonso Canales, Antonio Colinas, Carmen Conde, Ángel Crespo, Carlos de la Rica, Arturo del Villar, Gerardo Diego, Manuel Fernández Calvo, María de los Reyes Fuentes, Pablo García Baena, Ángel García López, Juan GilAlbert, José Luis Giménez-Frontín, Jorge Guillén, José Gutiérrez, Alfonso López Gradolí, Manuel Mantero, Joaquín Márquez, Enrique Molina Campos, Rafael Montesinos, Carlos Murciano, Fernando Ortiz, Rafael Pérez Estrada, Gregorio Prieto, Fernando Quiñones, Manuel Ríos Ruiz, Mariano Roldán, Juvenal Soto, José Luis Tejada y Rosendo Tello Aina.

Gerardo Diego aporta un texto en prosa, "La 'Égloga' de Cernuda”, que aparece al tiempo en el periódico Arriba (Diego, 1977, p. 13). En él rememora su amistad con el sevillano y la admiración por su poesía a partir de la petición que le hizo de algún original para el primer número de la revista Carmen, que finalmente se abre por decisión confesa de Diego con su "Égloga": "Y yo decidí, a la vista de los primeros originales llegados para el primer número de Carmen, que los presidiese el más importante a mi juicio, el más subido de calidad, 'Égloga"' (Diego, 1977, p. 13). Remata su evocación recordando el último encuentro entre ambos en México en 1958.

Por edad, ya que nace en 1904, y por amistades literarias, Juan Gil-Albert forma parte de la constelación del 27 aunque su arranque poético público sea algo más tardío. En este homenaje a Cernuda entrega el poema "La sed propia" (Gil-Albert, 1977, p. 19), recogido en la sección "Poemas sueltos" de su Poesía completa (GilAlbert, 2004, p. 935). Gil-Albert construye un poema en el que trae a colación un conjunto de escritores (Virgilio, Dante, Hölderlin, Baudelaire, Machado y Cernuda) para resaltar que ningún poeta puede saciar su sed a expensas y solo con la lectura de otros autores, sino que todo poeta tiene que beber de su propio manantial que se haya escondido dentro de sí. De Cernuda escribe a este respecto de lo que cada poeta ofrece para calmar la sed: "ni la copa / esbelta en que Cernuda un oro amargo / le tiende sin querer".

Jorge Guillén presenta un poema manuscrito titulado "Perfil del viento. En el homenaje a Luis Cernuda", que forma parte de sus libros Homenaje - Reunión de vidas - y Aire nuestro: Cántico, Clamor, Homenaje (Guillén, 1977, p. 22). Guillén dota su poema de una estructura dialogada para anotar algunas claves que explican el ser de Cernuda y de su poesía: la vaguedad, el alma dolorida, la altivez serena, el retiro, la soledad, la hermosura insinuada y el silencio.

Carmen Conde participa con el poema "A Luis Cernuda”, del que apunta a pie de página que forma parte del libro inédito Aynadamar y en el que parece abordar una cuestión amorosa en términos de ausencia, dolor, angustia, desamparo, silencio y soledad (Conde, 1977: 7).

\section{El homenaje a Vicente Aleixandre}

Ya en el número dedicado a Cernuda, de noviembre de 1977, Joaquín Márquez refiere la oportunidad del número por motivos cernudianos y, de paso, para celebrar el Premio Nobel que la Academia Sueca concede a Aleixandre el 6 de octubre de 1977. Solo unos meses más tarde, en mayo-junio de 1978, sale de imprenta el número doble 27-28 que lleva por título en su cubierta "A los ochenta años del poeta sevillano Vicente Aleixandre" y que rinde tributo, además de a un Premio Nobel, a un escritor sevillano que había colaborado con la revista Cal. En el número centrado en Cernuda figura el poema autógrafo de Jorge Guillén y quizás de ahí nace la idea de que el dedicado a Aleixandre lleve todos sus textos de forma autógrafa, lo que le concede una singularidad y vistosidad tipográficas muy sobresalientes. Una vez más en Cal se disponen las contribuciones siguiendo el orden alfabético de sus creadores: Rafael Alfaro, María Victoria Atencia, Alfonso Canales, José Luis Cano, Guillermo Carnero, Carmen Conde, Ángel Crespo, Miguel Fernández, María de los Reyes Fuentes, Ángel García López, José García Nieto, Jaime Gil de Biedma, Juan Gil-Albert, José Luis 
Giménez-Frontín, Jorge Guillén, Rafael Guillén, Manuel Jurado López, Manuel Mantero, Joaquín Márquez, Juan Mena, Enrique Molina Campos, Ana María Navales, Rafael Pérez Estrada, Fernando Quiñones, Carlos de la Rica, Pedro Rodríguez Pacheco, Onofre Rojano, José Ruiz Sánchez, Rafael Soto Vergés, José Luis Tejada, Julia Uceda, Pedro Luis Ugalde y Arturo del Villar. Según se aprecia en el listado, entre los escritores de la órbita del 27 que se dan cita se encuentran José Luis Cano, Carmen Conde, Juan Gil-Albert y Jorge Guillén.

José Luis Cano colabora con el poema "Miseria de la palabra" (Cano, 1978, p. 6), que forma parte de su libro Poemas crepusculares y que presenta diferencias muy grandes y reseñables -incluso con versos nuevos o muy cambiados- con respecto a la versión recopilada en su Poesía completa (Cano, 2001, p. 238). El autor cita un verso de Aleixandre, "La palabra, esa lana marchita", de su poema "Palabras", a modo de paratexto bajo la línea del título. De tal guisa que Cano propone un diálogo con Aleixandre en torno a la reflexión sobre el alcance, la vigencia y la caducidad de las palabras.

Carmen Conde participa con el poema "Academia", que dedica a "A Vicente, con mucho cariño" y que fecha el 10 de febrero de 1972 en Madrid (Conde, 1978, p. 8). Se adelanta así significativamente a 1978, en que fue elegida miembro de la Real Academia de la Lengua, pues en el poema recrea el proceder de los académicos a la hora de seleccionar, discutir, valorar las nuevas palabras de una lengua.

Juan Gil-Albert entrega el soneto titulado "La Alta Noche", que fecha en diciembre de 1951 y del que indica que es inédito (Gil-Albert, 1978, p. 18). No alude a Aleixandre directamente, pues consiste en un texto poético, no falto de juego barroco, en el que procura definir la noche a partir de la luz del día.

Jorge Guillén colabora con un poema muy breve que titula "En el homenaje a Vicente Aleixandre", del que señala que forma parte del libro en preparación Final (Guillén, 1978, p. 21). Se trata de un texto donde reflexiona sobre la creación, la inspiración, el arte y la vida a partir de una intertextualidad que remite a la idea de la musa torrencial de Eugenio Montale.

\section{LOS POEMAS SUELTOS}

Finalmente, cabe mencionar algunos poemas de escritores de la órbita del 27 que aparecen de forma suelta en los distintos números de Cal.

Rafael Laffón colabora en el número 1 con "Santidad doméstica”, un poema que dedica "A Gerardo Diego, por su espléndido ejemplar de Preludio, Aria y Coda que desde hace mucho tiempo me tiene dedicado" y que recrea algunas labores domésticas que se revisten de cierta santidad y espiritualidad (Laffón, 1974, p. 10). Concha Lagos contribuye con "Sin rumbo" en el número 1, un poema reflexivo y confesional en el que plantea algunas de sus dudas ante el mundo y declara su estado de soledad (Lagos, 1974, p. 11). José María Pemán escribe "Verso del mundo" en el número 2, un poema intimista en el que establece un puente de unión entre su estado sentimental apenado y el mundo externo (Pemán, 1974, p. 21). José Jurado Morales entrega tres "Poemas breves" para el número 3, también intimistas, en los que reflexiona sobre los motivos poéticos de la realidad externa y la capacidad creativa del poeta para asir esa realidad (Jurado Morales, 1974, p. 10). Juan Gil-Albert participa con "El primer día de otoño" en el número 6. Se trata de un poema fechado en setiembre de 1963 donde celebra la belleza del mundo primigenio y natural: la luz, la tierra, el sol, lo azul del cielo, etc.; "la bondad primigenia de las cosas", dice en un verso (Gil-Albert, 1974, pp. 8-9). Juan Ruiz Peña entrega el poema "Bécquer" para el número 30, un tributo agradecido a las enseñanzas recibidas del poeta sevillano (Ruiz Peña, 1978, p. 15). Elena Martín Vivaldi colabora con “Después del amor” en el número 17, donde reconstruye los sentimientos habidos después de un encuentro amoroso (Vivaldi, 1976, p. 10).

En definitiva, la revista Cal atesora el mérito de haber contribuido a la revitalización de la poesía sevillana y de haber dado cauce a la creación de poetas muy diversos en los tiempos de la Transición política de los años setenta. Con el paso de los años se aprecia mejor el propósito de Joaquín Márquez y sus compañeros de abrir un diálogo intergeneracional y apostar por la recuperación de algunas voces veteranas que ayudase a enlazar el pasado y el presente poético de Sevilla y, en general, de Andalucía. Desde esta óptica hay que 
valorar y leer los textos de los poetas del núcleo central del 27 incluidos en Cal: Vicente Aleixandre, Luis Cernuda, Gerardo Diego y Jorge Guillén, al igual que los de otros coetáneos que inician su trayectoria en la Edad de Plata: Juan Sierra, Rafael Laffón, José María Pemán, Manuel Barbadillo, José Jurado Morales, Juan Ruiz Peña, Ramón de Garciasol, Elena Martín Vivaldi, Gregorio Prieto, José Luis Cano, Juan Gil-Albert, Carmen Conde y Concha Lagos.

\section{REFERENCIAS}

AA.VV. (1960). Homenaje al poeta sevillano Rafael Laffón. Sevilla: Ayuntamiento.

Aleixandre, V. (mayo-julio de 1976). Retorno. Poetas de Sevilla, Cal. Revista de poesia (Sevilla), 14-15, 3.

Barbadillo, M. (mayo-julio de 1975). A Rafael Laffón. Homenaje a Rafael Laffón, Cal. Revista de poesía (Sevilla), 9, 13. Barrera, J. Ma (1992). La revista Cal. Historia e Índices. Revista de Humanidades (UNED, Sevilla), 3, 143-154.

Cano, J. L. (mayo-junio de 1978). Miseria de la palabra. A los ochenta años del poeta sevillano Vicente Aleixandre, Cal. Revista de poesia (Sevilla), 27-28, 6 .

Cano, José Luis (2001). Poesía completa, ed. A. Sanz, prólogo de L. de Luis. Algeciras: Fundación Municipal de Cultura José Luis Cano.

Cenizo Jiménez, J. (2002). Poesía sevillana: grupos y tendencias (1969-1980). Sevilla: Universidad.

Conde, C. (noviembre de 1977). A Luis Cernuda. Homenage [sic] a Luis Cernuda, Cal. Revista de poesía (Sevilla), 23-24, 7 .

Conde, C. (mayo-junio de 1978). Academia. A los ochenta años del poeta sevillano Vicente Aleixandre, Cal. Revista de poesia (Sevilla), 27-28, 8.

Cózar, R. de (1977). Cuatro capítulos de una historia. En AA.VV., Nueva poesia: Sevilla (pp. 25-27). Madrid: Zero, col. Guernica, 9.

Cruz Giráldez, M. (1994). Angaro (1969-1994). Veinticinco años de poesía en Sevilla (Estudio y selección). Sevilla: Ángaro, col. de Poesía, 116-117.

Diego, G. (1967). Segunda antología de sus versos (1941-1967). Madrid: Espasa-Calpe, col. Austral.

Diego, G. (mayo-julio de 1975). Primavera de Rafael Laffón. Homenaje a Rafael Laffón, Cal. Revista de poesía (Sevilla), 9,5.

Diego, G. (noviembre de 1977). La 'Égloga' de Cernuda. Homenage [sic] a Luis Cernuda, Cal. Revista depoesía (Sevilla), 23-24, p. 13. Reproducido en Arriba (Madrid), 5 de febrero de 1978, y en Diego, G. (2000). Obras completas (p. 530), ed. José Luis Bernal. Madrid: Alfaguara, 2000, t. 8.

Fuentes, Ma de los R. (mayo-julio de 1975). (del homenaje a Rafael Laffón, 1960). Homenaje a Rafael Laffón, Cal. Revista de poesia (Sevilla), 9, 4.

Garciasol, R. de (mayo-julio de 1975). ¡Salud, Rafael Laffón!. Homenaje a Rafael Laffón, Cal. Revista depoesía (Sevilla), 9, 18-19.

Gil-Albert, J. (noviembre de1974). El primer día de otoño. Cal. Revista de poesía (Sevilla), 6, 8-9.

Gil-Albert, Juan (noviembre de 1977). La sed propia. Homenage [sic] a Luis Cernuda, Cal. Revista de poesia (Sevilla), 23-24, 19.

Gil-Albert, J. (mayo-junio de 1978). La Alta Noche. A los ochenta años del poeta sevillano Vicente Aleixandre, Cal. Revista de poesia (Sevilla), 27-28, 18.

Gil-Albert, J. (2004). Poesía completa, ed. María Paz Moreno Páez, introd. de Ángel Luis Prieto de Paula. Valencia: Pre-Textos \& Instituto Alicantino de Cultura Juan Gil-Albert.

Guillén, J. (noviembre de 1977). Perfil del viento. En el homenaje a Luis Cernuda. Homenage [sic] a Luis Cernuda, Cal. Revista depoesía (Sevilla), 23-24, 22. Incluido en Guillén, J. (1967). Homenaje - Reunión de vidas-. Milán: All'Insegna del Pesce d'Oro, y en Guillén, J. (1968). Aire nuestro: Cántico, Clamor, Homenaje. Milán: All'Insegna del Pesce d'Oro. 
Guillén, J. (mayo-junio de 1978). En el homenaje a Vicente Aleixandre. A los ochenta años del poeta sevillano Vicente Aleixandre, Cal. Revista de poesia (Sevilla), 27-28, 21.

Jiménez Martos, L. (mayo de 1978). Tres poetas del Sur. Estafeta literaria (Madrid), 635.

Jurado López, M. (noviembre de 1979). Linealidad poética entre Mallarmé, Cernuda y Juan Bernier. Cal. Revista de poesia (Sevilla), 36, 22-25.

Jurado López, M. (1980). La poesía sevillana de los años setenta (aproximación y análisis). Sevilla: Barro, col. Vasija, 9.

Jurado López, M. (4 de mayo de 1989). La desaparecida revista de poesía sevillana Cal. Cuadernos del Sur (Málaga), 112, VII-43.

Jurado López, M. (diciembre de 1994). Crónica de la poesía en Sevilla (Apuntes de los setenta). Zurgai (Bilbao), 20-24.

Jurado Morales, J. (mayo de 1974). Poemas breves. Cal. Revista de poesía (Sevilla), 3, 10.

Jurado Morales, J. (mayo-julio de 1975). A Rafael Laffón. Homenaje a Rafael Laffón, Cal. Revista de poesía (Sevilla), 9, 22.

Lagos, C. (enero de 1974). Sin rumbo. Cal. Revista de poesía (Sevilla), 1, 11.

Laffón, R. (enero de 1974). Santidad doméstica. Cal. Revista de poesía (Sevilla), 1, 10.

Laffón, R. (mayo-julio de 1975). Microantolología de Rafael Laffón. Homenaje a Rafael Laffón, Cal. Revista de poesía (Sevilla), 9, 6-10.

Laffón, R. (mayo-julio de 1976). Ni línea, ni color... Cal. Revista de poesía (Sevilla), 14-15, 4.

Linares, A. (setiembre de 1978). Una de Cal y otra de Aire. Tierras del Sur, 103, 53.

Márquez, J. (enero de 1974). Pórtico. Cal. Revista de poesía (Sevilla), 1, 2.

Márquez, J. (mayo-julio de 1976). Poetas de Sevilla. Cal. Revista de poesía (Sevilla), 14-15, 2.

Márquez, J. (noviembre de 1977). Sevilla 1977 (calle del aire). Homenage [sic] a Luis Cernuda, Cal. Revista de poesía (Sevilla), 23-24, 44.

Márquez, J. (setiembre de 1978a). Una de Cal y otra de Aire. Tierras del Sur, 103, 52.

Márquez, J. (noviembre de 1978b). Cal. Revista de poesia (Sevilla), 30, 2.

Martín Vivaldi, E. (noviembre de 1976). Después del amor. Cal. Revista depoesia (Sevilla), 17, 10.

Molina Campos, E. (noviembre de 1977). Cernuda 'en’ Hölderlin. Homenage [sic] a Luis Cernuda, Cal. Revista de poesia (Sevilla), 23-24, 27-29.

Molina Campos, E. (enero de 1978). Dos grandes poetisas andaluzas: Elena [Martín Vivaldi] y Julia [Uceda]. Cal. Revista de poesia (Sevilla), 25, 24-27.

Molina Campos, E. (mayo-junio de 1979). Los poetas de 'Silene’ y la poesía de José Gutiérrez. Cal. Revista de poesía (Sevilla), 33-34, 32-35.

Núñez, J. L. (mayo-julio de 1975). Razón del Homenaje. Homenaje a Rafael Laffón, Cal. Revista de poesia (Sevilla), 9,2-3.

Pemán, J. Ma (marzo de 1974). Verso del mundo. Cal. Revista de poesia (Sevilla), 2, 21.

Prieto, Gregorio (1981). Cernuda en línea. Madrid: Biblioteca Nueva.

Rodríguez Pacheco, P. y Sánchez Menéndez, J. (1992). Joaquín Márquez. En Poesía sevillana 1950-1990 (Estudio y Antología) (pp. 72-73). Brenes (Sevilla): Muñoz Moya y Montraveta editores.

Ruiz-Copete, J. de D. (1971). Poetas de Sevilla. De la generación del 27 a los taifas del cincuenta y tantos. Sevilla: Caja de Ahorros Provincial de San Fernando.

Ruiz-Copete, J. de D. (1983). Panorama poético de Sevilla (De las brumas del medievo a las postrimerias del XX). Sevilla: Barro, Vasija Colección de libros, 20.

Ruiz Noguera, Fo (noviembre de 1989). Sobre las últimas generaciones poéticas de Andalucía. Canente, 6, 53-62.

Ruiz Peña, J. (mayo-julio de 1975). Romance del andaluz. Homenaje a Rafael Laffón, Cal. Revista de poesía (Sevilla), $9,31$.

Ruiz Peña, J. (noviembre de 1978). Bécquer. Cal. Revista de poesía (Sevilla), 30, 15. 
Sierra, J. (mayo-julio de 1976). Canción de la mujer pública. Poetas de Sevilla, Cal. Revista de poesía (Sevilla), 14-15, 5. Simón Guzmán, F. (2008). La poesía andaluza de la transición (1966-1982): revistas y antologías (Tesis Doctoral). Universidad de Sevilla.

Simón Guzmán, F. (2015). Las revistas literarias andaluzas de la transición: una aproximación a los procesos de transducción en una cultura extrasistémica, 1968-1982. Sevilla: Centro Andaluz del Libro.

\section{Notas}

1 Este artículo se halla vinculado al Proyecto de Investigación del Plan Estatal "Poéticas de la Transición (1973-1982)" financiado por: FEDER/Ministerio de Ciencia, Innovación y Universidades- Agencia Estatal de Investigación/ FFI2017-84759-P.

2 Hay depositada una fotocopia en color de la carta que incluye "Jardín antiguo" en la Fundación Gregorio Prieto: https ://gregorioprieto.org/archivo/poema-jardin-antiguo-de-luis-cernuda-dedicado-a-gregorio-prieto/

3 Manuscrito depositado en la Fundación Gregorio Prieto: https://gregorioprieto.org/archivo/poema-el-amigo-de-luiscernuda-dedicado-a-gregorio-prieto/ 